\title{
Preliminary report on the pathogenicity of Legionella pneumophila for freshwater and soil amoebae
}

\author{
TIMOTHY J ROWBOTHAM \\ From the Leeds Regional Public Health Laboratory, Bridle Path, York Road, LS15 7TR, UK
}

SUMMARY Legionella pneumophila, the causative organism of Legionnaires' disease, is pathogenic for free living, ubiquitous, freshwater, and soil amoebae of the genera Acanthamoeba and Naegleria. Some species support the growth of strains from serogroups 1 to 6, others only strains from certain serogroups. Initial studies with seeded material indicate that amoebal enrichment could be utilised for the isolation of legionellae from clinical specimens and natural habitats. It is suggested that a vacuole, or amoeba, full of legionellae, rather than free legionellae, could be the infective particle for man.

Legionella pneumophila, the causative organism of Legionnaires' disease, has been isolated from the water of cooling towers, ${ }^{1-3}$ evaporative condensers, ${ }^{2} 4$ ponds, ${ }^{5}$ a thermally polluted stream, the mud of that stream, ${ }^{2}$ and various other aquatic habitats. It will survive several months, or longer, in water for injection, ${ }^{6}$ and in distilled, ${ }^{7} 8$ tap, ${ }^{7}$ stream, ${ }^{9}$ and pond water. ${ }^{10}$ However, laboratory media for the growth of L.pneumophila require supplementation with L-cysteine and ferric iron, usually ferric pyrophosphate. The main carbon sources for L.pneumophila are L-amino acids. Arginine, cysteine, isoleucine, leucine, methionine, threonine, valine, and phenylalanine or tyrosine are required for growth. Most strains require serine, some also require proline. Serine and, to a lesser extent, threonine can be used as sole sources of carbon and energy. ${ }^{11} 12$ Carbohydrates $^{11} 13$ and many organic acids are not utilised, while some organic acids, for example, citric and oleic acid, are inhibitory. ${ }^{11}$ Growth on solid media is initiated only within a narrow $\mathrm{pH}$ range, around $6.9(6 \cdot 3-7 \cdot 28) .1415$ The optimum incubation conditions are $35^{\circ} \mathrm{C}$ in air $+2.5 \%(\mathrm{v} / \mathrm{v})$ $\mathrm{CO}_{2}$; no growth occurs anaerobically. The temperature range over which growth can occur is approximately $25^{11}-40^{\circ} \mathrm{C}$. On first isolation, growth is usually very slow; only pin-point colonies are seen after three days. Growth of L.pneumophila on solid media is inhibited by many bacteria, for example, certain members of the oral flora, a Bacillus species ${ }^{16}$ and Pseudomonas aeruginosa, also by a purified pyocin (unpublished data). These cultural characteristics indicate that L.pneumophila

Received for publication 26 July 1980 is not a free living aquatic bacterium. Recently, a relationship between L.pneumophila and a Fischerella sp. has been described. Growth of L.pneumophila occurred only when the cyanobacteria were photosynthesising. ${ }^{17}$

In man, L.pneumophila infects macrophages, in which large numbers of legionellae have been observed intracellularly. ${ }^{18-20}$ Extracellular legionellae appear to be closely associated with lysed macrophages. ${ }^{18}$ In-vitro studies with cynolmolgus monkey macrophages ${ }^{21}$ showed that L.pneumophila can grow in their cytoplasm and that, although rat and mouse alveolar macrophages ingest only antibody-coated L.pneumophila, ${ }^{22}$ this is not a necessary prerequisite for phagocytosis by cynolmolgus monkey macrophages. ${ }^{21}$ Intracellular L.pneumophila in polymorphonuclear leucocytes appears to be rare. ${ }^{18} 23$ Macrophages are amoeboid cells and, because of the similarity between macrophages and amoebae, this study was initiated to determine the interactions of L.pneumophila and similar organisms with amoebae. Free living amoebae of the genera Naegleria and Acanthamoeba are ubiquitous in soil and water. The commonest species, A.polyphaga, A.castellanii, and N.gruberi, have been isolated from humidifiers and may be present there in large numbers. ${ }^{24}$

\section{Methods}

\section{CULT URE}

Ninety millimetre diameter plates of Stoianovitch's malt extract, yeast extract agar, ${ }^{25}$ prepared with Page's amoebal saline, ${ }^{25} \mathrm{pH} 6.9$, were flooded with a turbid suspension of Klebsiella aerogenes (NCTC 
7427) in amoebal saline, drained, and incubated aerobically overnight at $35^{\circ} \mathrm{C}$. The agar was divided into two semicircular sections by the removal of a diagonal $10 \mathrm{~mm}$ wide strip. To kill the klebsiellae, the open plates were exposed for 30 minutes to the UV light of a microbiological safety cabinet. One end of each semicircular lawn was inoculated with a suspension of amoebal trophozoites, and approximately $15-20 \mathrm{~mm}$ away a line of L.pneumophila was drawn with a wire across the semicircle so that the advancing amoebae would have to cross it. The wire was inoculated with legionellae from an enriched blood agar ${ }^{26}$ (with soluble starch $1.5 \mathrm{~g} / \mathrm{l}$, and $\mathrm{L}$-serine $1.0 \mathrm{~g} / \mathrm{l}$ ) plate, incubated at $35^{\circ} \mathrm{C}$, in air $+2.5 \% \mathrm{v} / \mathrm{v} \mathrm{CO}_{2}$, for three to five days. On the control side, UV-killed klebsiellae were used. Amoebal cultures were incubated aerobically at $30^{\circ} \mathrm{C}$, the sump temperature of the type of humidifiers from which L.pneumophila has been isolated. ${ }^{27}$

\section{MICROSCOPY}

The amoebae were examined microscopically in situ on the agar surface with a $\times 4012 \mathrm{~mm}$ working distance objective (Vickers Instruments, Haxby Road, York). Isolation of single cysts and amoebae was facilitated by the long working distance of this objective and the absence of image inversion and reversion. For higher magnifications, amoebae were suspended in one drop of amoebal saline on a $22 \times 64 \mathrm{~mm}$ cover slip. Small drops of glycerol were placed at each end, and a cavity slide was inverted over the cover slip. The mount was left in that position for at least $\mathbf{2 0}$ minutes to allow the amoebae to extend and adhere to the cover slip; the slide was inverted for examination. After examination of the live material the slide was removed, and 3 drops of hot $\left(\sim 80^{\circ} \mathrm{C}\right) 10 \%(v / v)$ formalin were added to the amoebal drop just before it dried. After 2 minutes the cover slip was immersed for 2 minutes in unheated $10 \%(\mathrm{v} / \mathrm{v})$ formalin, rinsed in tap water, air dried, and stained by a modification of the Giménez technique, ${ }^{28}$ in which the buffered carbol fuchsin was warmed to $50^{\circ} \mathrm{C}$, filtered on to the cover slip, and left for 10 minutes. After thorough washing in tap water, the cover slip was stained with aqueous malachite green $(8 \mathrm{~g} / \mathrm{l})$ for 5-10 seconds, washed in tap water, stained again with the malachite green for 5-10 seconds, washed in tap water, and finally stained with aqueous methylene blue $(40 \mathrm{mg} / \mathrm{l})$ for $60-90$ seconds, washed, and dried. With this technique klebsiellae (coccobacilli) were blue to pale pink, rarely maroon, legionellae (bacilli) pink to maroon, amoebal cytoplasm pale blue, and nucleus blue-grey. The identity of the extracellular bacteria can be con- firmed by immunofluorescence but not of those intracellularly, as antibody does not enter intact amoebae.

\section{Results and discussion}

The Oxford-129-31 strain of L.pneumophila isolated from a patient in an Oxford renal transplant unit ${ }^{29}$ was chosen for special study because it was a recent isolate and thus less likely to be laboratory-adapted, and because similar strains had been isolated from the sieves of the transplant unit showers (JO'H Tobin and RG Mitchell, personal communication). The slowest growing amoeba, Acanthamoeba castellanii, took about five days to reach the line of legionellae. After two days in contact with the line there was no advance of the amoebae beyond the line. The amoebae in contact with the line were usually rounded up, smaller, and vacuolate; some were lysed. Heavily infected amoebae (Fig. 1)

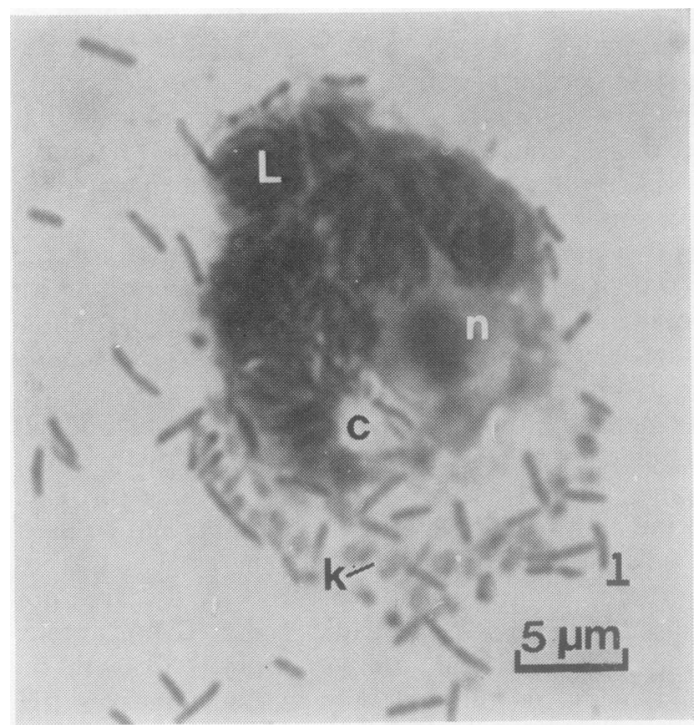

Fig. 1 A.castellanii trophozoite infected with the Oxford-1 strain of L.pneumophila. Modified Giménez stain. $L=$ endoplasmic vacuole containing legionellae; $n=$ nucleus; $c=$ contractile vacuole $; k=$ extracellular klebsiellae; $\quad l=$ extracellular legionellae around the amoeba.

occurred in the vicinity of the line. The infection was confined to vacuoles, often up to 10-15 vacuoles/amoeba. The legionellae in the vacuoles were non-motile. In some rare amoebae, the infection was not confined, and these amoebae developed into bags of non-motile legionellae. This latter type of infection was more common with the other 
L.pneumophila strains and is similar to that seen in human ${ }^{18}$ and monkey ${ }^{21}$ macrophages. In preparations of live material, some amoebae contained numerous large vacuoles, but even heavily infected amoebae usually produced pseudopodia. On the control side, the amoebae advanced beyond the line of dead klebsiellae; no legionellae could be demonstrated in those amoebae. Small groups of legionellae and single legionella in recently infected amoebae could be demonstrated only by staining. Some amoebae were so heavily infected (Fig. 1) that the infection could only be fatal. Infected amoebae were easily ruptured. Similar results were obtained with Oxford-1 and A.polyphaga.

An Acanthamoeba sp. isolated from a local humidifier grew much faster and encysted more rapidly than the axenically maintained culture collection acanthamoebae. Growth across the entire plate occurred in three to four days and was not prevented by the line of legionellae, which were phagocytosed. Numerous faecal vesicles, $2-4 \mu \mathrm{m}$ in diameter, were present in the area of the legionella inoculum; these did not contain recognisable legionellae. A heavy lawn of mature cysts was laid down on the inoculum line. Particularly where the inoculum was heavy, cysts were present before and after the line. On the control side, many scattered cysts were present, but few were arranged on the position of the klebsiella inoculum line. On the test side, after 48 hours malformed cysts, some empty, and grotesquely malformed amoebae were seen behind the line and between the line and the advancing edge of amoebal growth. No cysts or malformed amoebae were seen at the advancing edge of amoebal growth. In hanging drop preparations, the amoebae usually appeared normal, but some rare amoebae had a single large endoplasmic vacuole containing many rapidly motile bacilli. These infected amoebae were still moving, and apparently feeding, explaining how infected amoebae and failed cysts could be found behind the inoculum line. Large vesicles presumably from ruptured or lysed amoebae were rare, but some of these also contained rapidly moving bacilli. When stained, some of the smaller amoebae were also found to be infected. The results for other strains are given in the Table. In some rare amoebae, one to three intracellular filaments were seen; occasionally a filament would be almost coiled. Similar coiled forms of legionella have been reported in human lung. ${ }^{32}$ Mature cysts appear to be free from, and resistant to, infection. No legionellae were observed intracellularly in the flagellate phase of Naegleria. Studies on the temperature limits for infection are still in progress, but at room temperature $\left(\sim 21^{\circ} \mathrm{C}\right)$ it is more difficult to find infected amoebae.

Amoebae of the genera Acanthamoeba and Naegleria are ubiquitous in soil and fresh water. These studies show that they are possible natural hosts for L.pneumophila. The addition of UV-killed klebsiellae to humidifier sediments at room temperature greatly increased the number of amoebal

\begin{tabular}{|c|c|c|c|c|c|c|c|c|c|c|c|}
\hline \multirow[t]{2}{*}{$\begin{array}{l}\text { L.pneumophila } \\
\text { strain }\end{array}$} & \multirow[t]{2}{*}{ Serogroup } & \multicolumn{2}{|c|}{$\begin{array}{l}\text { Acanthamoeba } \\
\text { castellanii } \\
\text { AC L1501/2A }\end{array}$} & \multicolumn{2}{|c|}{$\begin{array}{l}\text { Acanthamoeba } \\
\text { polyphaga } \\
\text { AP L1501/3A }\end{array}$} & \multicolumn{2}{|c|}{$\begin{array}{l}\text { Acanthamoeba } \\
\text { sp. humidifier } \\
\text { strain** }\end{array}$} & \multicolumn{2}{|c|}{$\begin{array}{l}\text { Naegleria } \\
\text { gruberi } \\
1518 / 1 E\end{array}$} & \multicolumn{2}{|c|}{$\begin{array}{l}\text { Naegleria } \\
\text { jadini } \\
\text { B1518/2 }\end{array}$} \\
\hline & & $C P E$ & $I$ & $C P E$ & $I$ & $C P E$ & $I$ & $C P E$ & $I$ & $C P E$ & $I$ \\
\hline $\begin{array}{l}\text { Philadelphia-1 } \\
\text { NCTC } 11192\end{array}$ & 1 & +++ & +++ & +++ & +++ & C & FV & C & $\mathbf{F V}$ & C & FV \\
\hline $\begin{array}{l}\text { Philadelphia-2 } \\
\text { NCTC } 11193\end{array}$ & 1 & +++ & +++ & +++ & +++ & C & FV & C & FV & C & FV \\
\hline $\begin{array}{l}\text { Pontiac-1 } \\
\text { NCTC } 11191\end{array}$ & 1 & ++ & +++ & +++ & $++t$ & C & FV & C & FV & C & FV \\
\hline Nottingham-8 & 1 & +++ & $\underset{\mathrm{m}}{++}+$ & +++ & +++ & $\mathrm{C}$ & FV & C & FV & C & FV \\
\hline Togus-1 & 2 & +++ & +++ & +++ & +++ & +++ & $\underset{\mathrm{m}}{+++}$ & +++ & +++ & +++ & $\underset{\mathrm{m}}{++}$ \\
\hline $\begin{array}{l}\text { Bloomington-2 } \\
\text { Los Angeles-1 } \\
\text { Cambridge-2 } \\
\text { Oxford-1 }\end{array}$ & $\begin{array}{l}3 \\
4 \\
5 \\
6\end{array}$ & $\begin{array}{l}+++ \\
+++ \\
+++ \\
+++\end{array}$ & $\begin{array}{l}+++ \\
+++ \\
++ \\
+++\end{array}$ & $\begin{array}{l}+++ \\
+++ \\
+++ \\
+++\end{array}$ & $\begin{array}{l}+++ \\
+++ \\
++ \\
+++\end{array}$ & $\begin{array}{l}+t+ \\
\mathbf{C} \\
+++ \\
+++\mathrm{C}\end{array}$ & $\begin{array}{l}+++ \\
\text { FV } \\
++ \\
+ \text { FV } \\
\text { Mm }\end{array}$ & $\begin{array}{l}\stackrel{+}{+}+ \\
\stackrel{+}{\mathbf{C}}\end{array}$ & $\begin{array}{l}+++ \\
\text { FV } \\
++ \\
\text { FV }\end{array}$ & $\begin{array}{l}++ \\
\text { C } \\
\stackrel{+}{\mathbf{C}}\end{array}$ & $\begin{array}{l}+++ \\
\text { FV } \\
+++ \\
\text { FV }\end{array}$ \\
\hline
\end{tabular}

*Clone derived from a single cyst. CPE $=$ cytopathic effect (rounding up, vacuolation, lysis): + Slight $;++$ Moderate; +++ Marked; advance of amoebae often stopped by the legionella inoculum line; + Grotesquely deformed amoebae; $\mathrm{C}=$ an increase in the number of cysts on the legionella inoculum line, particularly where the inoculum was heavy.

$\mathbf{I}=$ infection; + rare; ++ several; +++ most amoebae; $\mathbf{F V}=$ large numbers of faecal vesicles on the legionella inoculum line; $\mathbf{M}=$ legion$\mathrm{I}=$ infection; + rare; ++ several; +++ most amoebae; $\mathrm{FV}=$ large numbers of faecal vesicles on the legionella inoculum line; $\mathrm{M}=1$ ex
ellae motile inside an endoplasmic vacuole of a live amoeba, rare; $\mathrm{m}=$ legionellae motile inside an extracellular vesicle, uncommon.

Named amoebae from the Culture Collection of Algae and Protozoa, Cambridge; NCTC = National Collection of Type Cultures, Colindale, London. 
trophozoites. Rotifers, if present, selectively grazed these (Fig. 2) until only rare amoebal cysts were left; the rotifers themselves then declined. A further addition of dead klebsiellae enabled the cycle to be repeated. These initial studies show that a population of suitable feeding amoebae at a temperature above $21^{\circ} \mathrm{C}$, probably in the region of $30-35^{\circ}$, would be necessary for large numbers of legionellae to occur in a humidifier. Cooling towers and evaporative condensers, implicated so often in Legionnaires' disease outbreaks, ${ }^{33}$ operate at, or near, the optimal growth temperatures for amoebae and L.pneumophila. Infected amoebae are fragile and thus likely to burst when forced through the spray nozzles of such equipment. With the continual recycling of the water, most amoebae could quickly become infected. Antifouling chemicals might inhibit rotifers, and filters used to prevent the spray nozzles from clogging may also remove them, allowing the amoebal population to increase above the levels found in natural habitats. Infected amoebae and vesicles containing legionellae would be present in the drift from contaminated equipment. The larger amoebae might be caught by the baffle plates, but smaller amoebae and vesicles would be less likely to be removed.

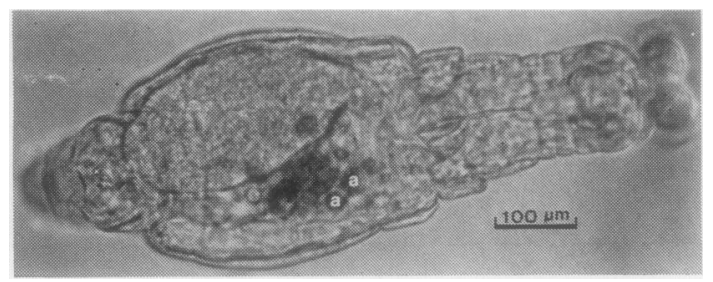

Fig. 2 Live rotifer feeding on amoebae (a). From this material out of a local humidifier the Acanthamoeba sp. in the Table was isolated. A trace of methylene blue was added to the preparation to stain the amoebae.

It is conceivable that infection is acquired not by inhaling free legionellae but by inhaling a vesicle or an amoeba full of legionellae. The potential host would then receive up to 50 or, in the case of an amoeba, probably up to $\mathbf{1 0 0 0}$ or more legionellae at once. The surrounding vesicle membrane, or amoeba, would help to prevent rapid dehydration of the legionellae. Such a mechanism would explain the lack of patient spread in Legionnaires' disease and the occurrence of the Broad Street cases in the 1976 Philadelphia outbreak, ${ }^{34}$ and the four cases in people who passed by in an adjacent street to a Memphis hospital during an outbreak. ${ }^{3}$ Trials with seeded material suggest that amoebal enrichment could perhaps be utilised for the recovery of legion- ellae from natural habitats and clinical specimens. Certain serogroups might be selectively enriched (Table). The WIGA (ALLO-1) ${ }^{35}$ strain of Legionella bozemanii, ${ }^{36}$ and the legionella-like organism Tex$\mathrm{KL}^{37}$ isolated from cases of fatal human pneumonia, also infect amoebae (in preparation). Further studies, including electron microscopy, on the interactions of $L$.pneumophila and similar organisms with amoebae are in progress.

I am indebted to Dr LR Hill, of the NCTC, for the WIGA, Tex-KL, and NCTC L.pneumophila strains, and to Mr PG Greaves, of the Nottingham Public Health Laboratory, for the other legionella strains.

\section{References}

${ }^{1}$ Morris GK, Patton CM, Feeley JC, et al. Isolation of the Legionnaires' disease bacterium from environmental samples. Ann Intern Med 1979;90: 664-6.

${ }^{2}$ Morris GK, Skaliy P, Gorman GW, et al. Environmental sources of Legionella pneumophila, the etiologic agent of Legionnaires' disease. Abstracts of the Annual Meeting of the American Society for Microbiology 1979;326.

${ }^{3}$ Dondero TJ Jr, Rendtorff RC, Mallison GF, et al. An outbreak of Legionnaires' disease associated with a contaminated airconditioning cooling tower. $N$ Eng $J$ Med 1980;302:365-70.

4 Cordes LG, Fraser DW, Skaliy P, et al. Legionnaires' disease outbreak at an Atlanta, Georgia, country club: evidence for spread from an evaporative condenser. Am J Epidemiol 1980;111:425-31.

5 Fliermans CB, Cherry WB, Orrison LH, Thacker L. Isolation of Legionella pneumophila from nonepidemicrelated aquatic habitats. Appl Environ Microbiol 1979; 37:1239-42.

6 Highsmith AK, Mackel DC, Anderson RL, et al. Resistance of Legionella pneumophila in sterile water for injection. Abstracts of the Annual Meeting of the American Society for Microbiology 1979;231.

'Skaliy P, McEachern HV. Survival of the Legionnaires' disease bacterium in water. Ann Intern Med 1979;90: 662-3.

${ }^{8}$ Durham LC, Miller RD. Physical stability and survival of Legionnaires' disease bacterium (Legionella pneumophila) in an aqueous environment. Abstracts of the Annual Meeting of the American Society for Microbiology, 1979;50.

9 Wang WLL, Blaser ML, Cravens J, Johnson MA. Growth, survival, and resistance of the Legionnaires' disease bacterium. Ann Intern Med 1979;90:614-8.

10 Janssen WA, Larson RG. Studies on the survival and growth of Legionnaires' disease bacteria in vitro. Abstracts of the Annual Meeting of the American Society for Microbiology 1979;50.

${ }^{11}$ Pine L, George JR, Reeves MW, Harrell WK. Development of a chemically defined liquid medium for growth of Legionella pneumophila. J Clin Microbiol 1979;9:615-26.

12 George JR, Pine L, Reeves MW, Harrell WK. Amino acid requirements of Legionella pneumophila. J Clin Microbiol $1980 ; 11: 286-91$.

${ }^{13}$ Brenner DJ, Steigerwalt AG, McDade JE. Classification of the Legionnaires' disease bacterium: Legionella pneumophila, genus novum, species nova, of the family 
Legionellaceae familia nova. Ann Intern Med 1979;90: 656-8.

${ }^{14}$ Feeley JC, Gorman GW, Weaver RE, Mackel DC, Smith HW. Primary isolation media for Legionnaires' disease bacterium. J Clin Microbiol 1978;8:320-5.

15 Rutter DA, Maber HB. Culture of Legionella pneumophila. Lancet $1979 ; 1: 723-4$.

${ }^{16}$ Carrington GO. Legionnaires' disease bacillus: inhibition by normal flora. Clin Microbiol Newsletter 1979;1(9):7-8.

17 Tison, DL, Pope DH, Cherry WB, Fliermans CB. Growth of Legionella pneumophila in association with blue-green algae (Cyanobacteria). Appl Environ Microbiol 1980;39: 456-9.

${ }^{18}$ Chandler FW, Cole RM, Hicklin MD, Blackmon JA, Callaway CS. Ultrastructure of the Legionnaires' disease bacterium. A study using transmission electron microscopy. Ann Intern Med 1979;90:642-7.

19 Glavin FL, Winn WC Jr, Craighead JE. Ultrastructure of lung in Legionnaires' disease. Observations of three biopsies done during the Vermont epidemic. Ann Intern Med 1979;90:555-9.

${ }^{20}$ Rogers FG. Ultrastructure of Legionella pneumophila. $J$ Clin Pathol 1979;32:1195-202.

${ }^{21}$ Kishimoto RA, Kastello MD, White JD, et al. In-vitro interaction between normal cynolmolgus monkey alveolar macrophages and Legionnaires' disease bacteria. Infect Immun 1979;25:761-3.

22 Johnson W, Pesanti E, Elliott J. Serospecificity and opsonic activity of antisera to Legionella pneumophila. Infect Immun 1979;26:698:704.

${ }^{23}$ Chandler FW, McDade JE, Hicklin MD, Blackmon JA, Thomason BM, Ewing EP Jr. Pathologic findings in guinea pigs inoculated intraperitoneally with the Legionnaires' disease bacterium. Ann Intern Med 1979; 90:671-5.

24 Humidifier fever. Report of MRC Symposium 1976 Thorax 1977;32:653-63

25 Page FC. An illustrated key to freshwater and soil amoebae with notes on cultivation and ecology. Freshwater Biological Assoc, Ambleside, Cumbria, England. Scientific Publication No. 34, 1976.

${ }^{26}$ Greaves PG, Sharp G, Macrae AD. Isolation of Legionella pneumophila. Lancet 1979;1:551-2.

27 Miller RP. Cooling towers and evaporative condensers. Ann Intern Med 1979;90:667-70.
${ }^{28}$ Giménez DF. Staining of rickettsiae in yolk-sac cultures. Stain Technol 1964;39:135-40.

29 Taylor AG, Harrison TG. Legionnaires' disease caused by Legionella pneumophila serogroup 3. Lancet 1979;2:47. [Author's note: Strain now recognised by Center for Disease Control, Atlanta, Georgia, USA, as serogroup 6.]

${ }^{30}$ Fallon RJ, Abraham WH. Legionnaires' disease caused by Legionella pneumophila serogroup 3. Lancet 1979;2: 304

[Author's note: Strain now recognised by Center for Disease Control, Atlanta, Georgia, USA, as serogroup 6.]

${ }^{31}$ Fisher-Hoch S, Hudson MJ, Thompson MH. Identification of a clinical isolate as Legionella pneumophila by chromatography and mass spectrometry of cellular fatty acids. Lancet 1979;2:323-5.

${ }^{32}$ Hernandez FJ, Kirby BD, Stanley TM, Edelstein PH. Legionnaires' disease. Postmortem pathologic findings of 20 cases. Am J Clin Pathol 1980;73:488-95.

${ }^{33}$ Broome CV, Fraser DW. Epidemiologic aspects of legionellosis. Epidemiol Rev 1979;1:1-16.

34 Fraser DW, Tsai TR, Orenstein W, et al. Legionnaires' disease: description of an epidemic of pneumonia. N Engl J Med 1977;297:1189-97.

${ }^{35}$ Cordes LG, Wilkinson HW, Gorman GW, Fikes BJ, Fraser DW. Atypical Legionella-like organisms: fastidious water-associated bacteria pathogenic for man. Lancet 1979;2:927-30.

36 Brenner DJ, Steigerwalt AG, Gorman GW, et al. Legionella bozemanii species nova: classification of a second species of Legionella associated with human pneumonia. Curr Top Microbiol Immunol 1980 In press.

${ }^{37}$ Lewallen KR, McKinney RM, Brenner DJ, et al. A newly identified bacterium phenotypically resembling, but genetically distinct from Legionella pneumophila: an isolate in a case of pneumonia. Ann Intern Med 1979; $91: 831-4$

Requests for reprints to: Dr TJ Rowbotham, Leeds Regional Public Health Laboratory, Bridle Path, York Road, Leeds LS15 7TR. 